Artigo de Reflexão/Ensaio

\title{
Realidade virtual imersiva nos Cuidados Paliativos: perspectivas para a Reabilitaçáo Total
}

\section{Immersive virtual reality in Palliative Care: prospects for Total Rehabilitation}

\author{
Breno Augusto Bormann de Souza Filho ${ }^{\mathrm{a}, \mathrm{b}}$ (D), Érika Fernandes Tritany ${ }^{\mathrm{c}, \mathrm{d}}$ (D) \\ ${ }^{a}$ Universidade de Pernambuco - UPE, Recife, PE, Brasil. \\ ${ }^{b}$ Academia Paralímpica Brasileira - APB/CPB, São Paulo, SP, Brasil. \\ 'Universidade Federal do Rio de Janeiro - UFRJ, Macaé, RJ, Brasil. \\ dUniversidade Federal do Rio Grande do Norte - UFRN, Natal, RN, Brasil.
}

Como citar: Souza Filho, B. A. B., \& Tritany, E. F. (2022). Realidade virtual imersiva nos Cuidados Paliativos: perspectivas para a Reabilitação Total. Cadernos Brasileiros de Terapia Ocupacional, 30, e3024. https://doi.org/10.1590/2526-8910.ctoARF22923024

\begin{abstract}
$\underline{\text { Resumo }}$
Introduçáo: A Realidade Virtual se apresenta como ferramenta promissora para aprimoramento de tecnologias em saúde e potencialização de intervençóes para a melhora da funcionalidade e qualidade de vida de pacientes e familiares que enfrentam doenças e/ou condiçốes progressivas ameaçadoras da vida. Objetivo: Este ensaio discute o papel promissor da Realidade Virtual Imersiva na reabilitaçẫo em Cuidados Paliativos e propóe o conceito de Reabilitação Total como possibilidade para ampliação da concepçáo de reabilitação atual. Método: Apresentamos reflexóes baseadas na teoria de Dor Total, própria dos Cuidados Paliativos, e a inserção das Novas Tecnologias na saúde, especialmente no âmbito da reabilitaçáo, por meio de documentos baseados na linha reflexiva que os autores pretendem submeter à apreciação e ao debate público. Resultados: É importante e promissor o papel da Realidade Virtual Imersiva em intervençôes de saúde, bem como a proposta conceitual de ampliação do conceito e compreensão de Reabilitação, cunhando o termo Reabilitação Total. Além disso, fomentou-se o processo reflexivo de debate sobre as possibilidades terapêuticas e suas inovaçóes. Conclusáo: A partir da Reabilitação Total, inovaçóes relativas ao cuidado em saúde, sejam tecnológicas e/ou das práticas clínicas, podem ser aprimoradas e disponibilizadas através de intervençốes em ambientes físicos e/ou virtuais, tendo como premissas para os processos de reabilitação a funcionalidade global e dignidade da pessoa humana, com açôes que envolvam as dimensôes física, social, psicológica e espiritual, tal como apresentado pelo conceito de Dor Total.
\end{abstract}

Palavras-chave: Realidade Virtual, Terapia de Exposição à Realidade Virtual, Cuidados Paliativos, Reabilitação. 


\section{$\underline{\text { Abstract }}$}

Introduction: Virtual Reality presents itself as a promising tool for improving health technologies and enhancing interventions to improve the functionality and quality of life of patients and families facing progressive diseases and/or lifethreatening conditions. Objective: This essay discusses the promising role of Immersive Virtual Reality in Palliative Care rehabilitation and proposes the concept of Total Rehabilitation as a possibility to expand the current conception of rehabilitation. Method: We present reflections based on the Total Pain theory, typical of Palliative Care, and the inclusion of New Technologies in health, especially in the field of rehabilitation, through documents based on the reflexive line that the authors intend to submit for consideration and public debate. Results: The role of Immersive Virtual Reality in health interventions is important and promising, as well as the conceptual proposal for expanding the concept and understanding of Rehabilitation, coining the term Total Rehabilitation. In addition, the reflective process of debate on therapeutic possibilities and their innovations was fostered. Conclusion: From Total Rehabilitation, innovations related to health care, whether technological and/or clinical practices, can be improved and made available through interventions in physical and/or virtual environments, having global functionality and human dignity as premises for rehabilitation processes, with actions that involve the physical, social, psychological and spiritual dimensions, as presented by the concept of Total Pain.

Keywords: Virtual Reality, Virtual Reality Exposure Therapy, Palliative Care, Rehabilitation.

\section{Introduçáo}

Realidade Virtual (RV) é definida como o uso da tecnologia computacional para criar, manter um ambiente e projetar a presença física de um usuário neste ambiente, permitindo sua interaçáo com o meio (Cipresso et al., 2018).

A RV surgiu na década de 60 e vem ganhando notoriedade pelo desenvolvimento e aperfeiçoamento de aplicativos e softwares que otimizam meios de interação dos usuários. Além disso, o aprimoramento de dispositivos, como óculos imersivos e câmeras $360^{\circ}$, e sua aplicabilidade em diversos setores, como educação, engenharia, treinamento militar, saúde e outros, estimulam seu crescimento (Matthews, 2018; Cipresso et al., 2018).

$\mathrm{Na}$ área da saúde, em particular na reabilitação, a RV tem se apresentado como uma ferramenta promissora no aprimoramento de tecnologias (Johnson et al., 2018) e potencialização de métodos e técnicas de intervençóes para melhora da qualidade de vida de pacientes e seus familiares, que enfrentam doenças e/ou condiçóes progressivas e/ou ameaçadoras da vida (Cho \& Lee, 2019; Izard et al., 2018; Ferguson et al., 2020; Kim et al., 2018).

A Terapia de Exposição à Realidade Virtual (TERV) é compreendida como uma técnica terapêutica, em ambiente virtual, a qual permite que o participante experimente uma sensação de presença em um ambiente computacional imersivo, tridimensional e interativo que minimiza o comportamento de anulação e facilita o envolvimento emocional (Gerardi et al., 2010). 
Neste contexto, estudos clínicos demonstram a eficácia da TERV no cuidado em saúde. Sua utilização vem sendo direcionada a doenças, condiçóes e sintomatologias, como tratamento da dor (aguda e crônica), para sua atenuação e/ou distração frente a terapias invasivas (Ahmadpour et al., 2019), fadiga (Ioannou et al., 2020), distúrbios do sono (Lee \& Kang, 2020), controle do estresse (Vaquero-Blasco et al., 2021), fobias, ansiedade e depressão (Felnhofer et al., 2019), reabilitação motora (Tieri et al., 2018), entre outros. Além disso, a TERV pode facilitar a compreensão e aceitação da doença por parte do paciente e seus familiares, bem como o trabalho de aspectos motivacionais para início e manutenção do tratamento (Hsieh, 2020), afirmação da vida e aceitação da morte como processo natural, além de estímulos à interação social, afetividade e espiritualidade (Murdoch \& Davies, 2017; Wijma et al., 2018).

Assim, é importante estimular a incorporação da TERV aos planos de cuidados que objetivem açôes para manutenção e ganho de reserva funcional, bem como retardo no declínio da funcionalidade global, impactando positivamente na qualidade de vida e dignidade dos simbiontes do cuidado (pacientes, familiares/cuidadores e equipe multiprofissional) (Hsieh, 2020; Nwosu et al., 2021).

Neste ínterim, os Cuidados Paliativos (CP) se destacam como arcabouço técnicocientífico necessário para orientação dos planos de cuidado. Os CP apresentam uma abordagem que visa à prevenção e alívio do sofrimento e à promoção de dignidade, melhor qualidade de vida e adaptação a doenças e/ou condiçóes progressivas para adultos e crianças vivendo com sérios problemas de saúde, agudos ou crônicos, complexos e/ou ameaçadores da vida, bem como para suas famílias (Gómez-Batiste \& Connor, 2017). Pauta-se no conceito de Dor Total de Cicely Saunders (Miccinesi et al., 2020) - que compreende que não apenas os componentes físicos da dor devem ser considerados, mas também as dimensões sociais, psicológicas e espirituais.

Nesse sentido, o objetivo deste trabalho é apresentar uma reflexão sobre perspectivas da Realidade Virtual Imersiva para a reabilitação de pacientes em Cuidados Paliativos e propor um conceito de reabilitação que considere as transformaçóes tecnológicas como promissoras ao cuidado em saúde e baseado, como premissa básica, na teoria de Dor Total, incorporando à reabilitação uma visão multidimensional da dor e sofrimento humano, apresentando, assim, o conceito de Reabilitação Total.

Para tanto, este ensaio foi construído com base na escolha de referências que buscam refletir o posicionamento e linha reflexiva dos autores, de acordo com a perspectiva da Dor Total (Miccinesi et al., 2020), cunhada por Cicely Saunders - considerada a precursora dos Cuidados Paliativos modernos — bem como conceitos mais recentes relacionados aos Cuidados Paliativos de Reabilitação, propostos por Rebecca Tiberi e endossados pela Organização Mundial da Saúde (OMS) (Tiberini \& Richardson, 2015; World Health Organization, 2018).

\section{Realidade Virtual Imersiva nos Campos da Saúde e Reabilitaçáo}

Mudanças nos perfis demográfico e epidemiológico das populações, com envelhecimento populacional e maior prevalência de condiçóes crônicas no perfil de morbimortalidade mundial, além de aumento progressivo nos custos em saúde, pela intensa focalização assistencial e privilégio a modelos de atenção hospital-centrados e voltados para o uso de tecnologias diagnósticas e terapêuticas de alta densidade 
tecnológica, vêm desencadeando, em diferentes países, mudanças no modelo de atenção à saúde para cuidados mais próximos ao território e estratégias inovadoras, como os Cuidados Intermediários. Assim, verifica-se espaço fértil para apresentação de metodologias que atendam às necessidades de saúde mundiais com qualidade, efetividade e eficiência, sobretudo na área da Reabilitação (Tieri et al., 2018).

Assim, a combinação do protocolo de reabilitação com o uso de novas tecnologias (incluindo robótica, interfaces computacionais do cérebro, estimuladores cerebrais não invasivos, neuropróteses, dispositivo wearable para análise de movimento, tablet para reabilitação neurológica e Realidade Virtual) pode propiciar melhores projeçóes de resultados para a reabilitação. Dentre essas tecnologias, uma quantidade crescente de evidências científicas aponta a Realidade Virtual Imersiva (RVI) como uma ferramenta promissora para potencialização de resultados em Reabilitação (Izard et al., 2018; Montana et al., 2019; Qian et al., 2020; Zhu et al., 2021).

A RVI apresenta, no campo da saúde, uma espécie de revolução que vai desde conceitos relacionados a formas de tratamento e cuidado, alteraçóes em métodos de intervenção e treinamento em diferentes especialidades como fisioterapia, psicologia, medicina, enfermagem, terapia ocupacional, educação física e outras (Izard et al., 2018; Cipresso et al., 2018).

A TERV é uma alternativa às terapias tradicionais, sendo compreendida como uma técnica de tratamento em ambiente computacional, virtual, tridimensional, interativo e imersivo, o qual permite ao participante experimentar uma sensação de presença, que pode reduzir o comportamento de evitação às terapêuticas e facilita o envolvimento emocional do paciente (Gerardi et al., 2010).

O conceito de "presença" é central para teorizar sobre ambientes virtuais avançados, como a RVI. Presença pode ser caracterizada como sentido de estar no centro da perspectiva da observação e, ao mesmo tempo, no centro de sua construção (Weber et al., 2021). Trata-se de um complexo sentimento psicológico de imersão na $\mathrm{RV}$ que envolve a sensação da presença física e a possibilidade de interagir e reagir como se o usuário estivesse no mundo real. Quanto maior o nível de realismo dos estímulos apresentados, mais a expectativa do usuário será congruente com a expectativa de realidade. Assim, "presença” pode ser compreendida como a ilusão perceptiva da não mediação.

Com a RVI, o profissional possui controle da intervenção planejada, limitado apenas por características do software utilizado no conteúdo da experiência (Gerig et al., 2018; Pardo et al., 2018). Assim, o profissional poderá oferecer um contexto seguro e privado para o paciente, facilitando sua adesão, engajamento e aderência. Ou seja, a mediação da experiência virtual por parte do profissional é de suma importância para o sucesso terapêutico (Johnson et al., 2018).

Porém, para que tais efeitos sejam evidenciados, precisam ser consideradas questóes relacionadas à fase de progressão da doença e/ou condição em que o paciente e/ou familiares/cuidadores se encontram (fase inicial, intermediária, avançada e/ou luto), às adaptaçóes fisiológicas individuais necessárias, às limitaçóes tecnológicas e às especificidades do uso da RVI para cada fase da vida.

O poder da RVI é sua relação imersiva e interativa, que trabalha para levar lembranças subconscientes à percepção consciente (Matthews, 2018). O potencial da RV é espantoso, especialmente quando relacionada à utilização de avatares e 
representaçóes digitais impulsionadas por açóes, geralmente em tempo real. Experimentos psicológicos apontam mudanças no comportamento após experiências virtuais com avatares. Essas mudanças incluem maior confiança, porque seus avatares apresentam características consideradas privilegiadas e almejadas pelos usuários, por exemplo, altura, aparência, ou melhor situação/capacidade funcional e de saúde (Blascovich \& Bailenson, 2011). Além disso, a incorporação da sombra corporal pode representar uma classe de estímulos de alta prioridade que atua "empurrando" a atenção para o próprio corpo, contribuindo para a recuperaçáo cognitiva e motora e fortalecendo o sentido de presença (Russo et al., 2017).

A RV se apresenta como um ambiente seguro que permite ao paciente explorar e testar hipóteses, assim como desenvolver habilidades de enfrentamento mais eficazes, fortalecendo funçóes psicológicas que se tornaram enfraquecidas ou desviadas pelo próprio processo de envelhecimento, ansiedade ou trauma intenso, além de adoecimento ou tratamentos (Gerardi et al., 2010; Matthews, 2018).

A TERV, portanto, pode se representar como um portal para a imaginação, sendo um espaço no qual os pacientes podem descompactar suas próprias memórias, imaginaçôes e experiências e criar sua própria realidade. Ao fazê-lo, pode-se modificar a realidade para a qual foram tratados, superar medos, traumas ou condições relacionadas ao estresse e outras condiçóes (Buyuk et al., 2021; Felnhofer et al., 2019; Gerardi et al., 2010). Os pacientes trazem suas próprias memórias e transformam um mundo de fantasia gerado por computador em um lugar que eles reconhecem, do passado ou presente, que despertam uma conexão com sua própria história.

É crescente a aplicação de sistemas de RV para a reabilitação de uma variedade de déficits resultantes de lesóes do sistema nervoso. Destaca-se a reabilitação de pacientes com sequelas de Acidente Vascular Encefálico, sobretudo relativo à funcionalidade das extremidades superiores (Cho \& Lee, 2019; Tieri et al., 2018). Vários sistemas de RV para a reabilitação de membros superiores foram desenvolvidos e testados, no mundo, seguindo diferentes métodos e conceitos terapêuticos: sistemas para treinar movimentos de alcance baseados na imitação de um instrutor virtual; baseados em dispositivos hápticos (dispositivos táteis, que fornecem feedback sensorial aos usuários quando tocam objetos virtuais); de treino de movimentos gerais por ensaio mental, imitação de movimentos do braço não-parético; entre outros (Tieri et al., 2018).

Evidencia-se que, após o treinamento de RV, uma reorganização cortical é observada ocasionada pela neuroplasticidade, na qual a ativação cortical é reorganizada de ativação contralesional (antes de RV) para ipsilesional (após RV); isto é, há uma mudança na organização cortical do membro afetado do hemisfério ipsilateral para o hemisfério contralateral após a intervenção com a RV (Jang et al., 2005). Isso se deve provavelmente ao fato de que a $\mathrm{RV}$ pode ter promovido a reorganização práticadependente resultante do aumento da quantidade de uso do membro afetado em tarefas motoras relevantes (Jang et al., 2005).

Além disso, a RV permite a realização de terapias de relaxamento e controle do estresse em pessoas em ambientes confinados ou isolados (como hospitais), principalmente quando combinada com preferências dos indivíduos para a criação e escolha dos ambientes virtuais (Anderson et al., 2017; Johnson et al., 2020; Perna, 2021). Outrossim, a TERV facilita ainda a compreensão e a aceitação da doença por parte do paciente e seus familiares, afirmação da vida e aceitação da morte, além de 
estímulos à interação social e espiritualidade (Murdoch \& Davies, 2017; Wijma et al., 2018; Hsieh, 2020; Nwosu et al., 2021).

Acreditamos que, assim como outras técnicas de cuidado multidimensional em Cuidados Paliativos, essa pode também ser extensiva para familiares/cuidadores e equipe multiprofissional, a fim de aliviar a sobrecarga gerada pelo processo do cuidar. Resguardando-se as proporçóes e singularidades da família e cuidadores, podemos evidenciar a aplicação e eficácia da Realidade Virtual relacionadas a sentimento de empatia, fortalecimento da interação social e de laços afetivos e, ainda, no importante trabalho do luto, bem como podem ocorrer impactos positivos na comunicação entre paciente, família e equipe multiprofissional (Nwosu et al., 2021; Wang et al., 2020).

Ganha também destaque o uso da RV no treinamento e aperfeiçoamento da técnica para realização de procedimentos (mais ou menos invasivos), comunicação de má notícia, além de sentimento de responsabilidade e empatia, objetivando, assim, sensibilização da equipe multiprofissional envolvida no processo de cuidado (Lee et al., 2020; Nwosu et al., 2021; Wang et al., 2020). Outrossim, estresse, alta carga de responsabilidade e sentimento de culpa pela morte são exemplos de aspectos que podem ser trabalhados com os profissionais de saúde com o uso da RV.

Ressaltamos que, para atingir, com eficiência, os resultados desejados, a utilização da RV deve ser norteada pela fase do cuidado em que paciente, familiar e equipe multiprofissional se encontram. Assim, é possível o diálogo entre a equipe multiprofissional, paciente e familiares para desenho do projeto terapêutico, em que a RV possa ter aplicabilidade ora como tratamento neoadjuvante, ora adjuvante, e até mesmo com possibilidades de tratamento principal, a depender das condiçóes clínicas e desejo dos envolvidos (Cortés-Pérez et al., 2020; Kim et al., 2020).

Além disso, uma vez definida e incluída a TERV no plano de cuidado, deve-se privilegiar a centralidade no paciente, cuja orientação se dá pelo projeto terapêutico desenhado e não apenas pela tecnologia. Uma quantidade única e genérica de experiência imersiva não servirá para todos, sendo necessário flexibilidade e singularidade nos tratamentos. O importante é a "dose" correta de RV para cada paciente, compreendendo que alguns podem precisar de mais realismo ou mais intervençóes em ambiente virtual, ao passo que, para outros, podem ser indicadas menos seçôes de RV e/ou estratégias diferentes, sem mediação computacional, como a mentalização.

Nesse sentido, não devemos perder de vista o que é verdadeiramente importante: manter e fortalecer o relacionamento terapêutico; aumentar o empoderamento, autonomia e independência do paciente e sua família; e melhorar a qualidade do atendimento e o acesso dos pacientes em Cuidados Paliativos.

Os Cuidados Paliativos, por sua vez, estão alicerçados dentro de um modelo de cuidados totais, ativos, integrais e legitimados pelo direito de morrer e enfrentar o processo de adoecimento com dignidade. Essa modalidade de assistência valoriza a cultura, a espiritualidade, as tradições, os desejos e as crenças que permeiam o percurso do cuidado e os processos de fim de vida do paciente, considerando também a família e a equipe multiprofissional responsável pela condução do cuidado em saúde (GómezBatiste \& Connor, 2017).

As áreas centrais dos Cuidados Paliativos buscam se estender muito para além do alívio dos sintomas físicos, mas integrar os aspectos físicos, psicológicos, sociais e espirituais das 
práticas de cuidados, possibilitando à pessoa uma preparação para a sua morte de forma tấo completa, complexa e construtiva quanto possível, o que pressupóe integraçáo entre as pessoas envolvidas (paciente/família/equipe multiprofissional de saúde), dotadas de um processo de comunicaçáo adequado em prol do controle dos sintomas de dor física, psicológica, social e espiritual - Dor Total (Miccinesi et al., 2020) - e superando grandes desafios do trabalho em equipe, tais como a gestão de conflitos de forma construtiva e criativa, em prol de um bem comum: o respeito pela autonomia e bem-estar da pessoa em Cuidados Paliativos (Gómez-Batiste \& Connor, 2017).

\section{O Conceito de Dor Total}

No que se refere à prevenção e controle de sintomas, é fundamental considerar "sintoma" como tudo aquilo que o paciente avalia como um problema (Miccinesi et al., 2020). A partir do conceito de Dor Total, é possível compreender que não são somente os componentes físicos da dor que devem ser considerados, abarcando também as dimensóes emocional, social e espiritual. Atualmente, expande-se tal conceito para quaisquer sintomas, ou seja, coloca-se como fundamental evidenciar o caráter individual e subjetivo dos sintomas, bem como a interação entre fatores biológicos, sensoriais, afetivos, cognitivos, comportamentais, sociais e culturais na determinação, interpretação e expressão dos sintomas apresentados (Gómez-Batiste \& Connor, 2017).

A prevenção e o controle de sintomas são aspectos centrais da assistência paliativa. Ainda que existam especificidades de cada área profissional, todos que atuam devem ser capazes de identificar sintomas e conhecer técnicas básicas de manejo e/ou seus encaminhamentos. Escuta, apoio e orientação aos familiares são, portanto, inerentes aos cuidados.

A dor é um sintoma que exerce grande impacto sobre a qualidade de vida, influenciando no humor, sono e atividades de vida diária (AVD), no que tange às AVD básicas, instrumentais e avançadas (Tiberini \& Richardson, 2015). Outros sintomas, como anorexia, depressão, ansiedade, constipação, disfagia, dispneia, astenia, podem igualmente acometer o paciente, afetando relaçóes sociais, familiares e de trabalho. Por este motivo, o controle dos sintomas possibilita que o indivíduo possa realizar o que considera importante, proporcionando uma redução do sofrimento, sobretudo em fase de fim de vida (Gómez-Batiste \& Connor, 2017).

$\mathrm{O}$ apoio à família também deve ser contemplado nos $\mathrm{CP}$, uma vez que ela compartilha do sofrimento com o paciente. Assim, o processo de cuidado se amplia para a rede de relaçóes familiares, existindo ainda preocupação com o processo de luto, antes e após o óbito. As vantagens dos CP se pautam na possibilidade de melhor enfrentamento da morte, promovendo sua aceitação e minimizando o sofrimento físico e psicológico, tanto do paciente como de sua família (Gómez-Batiste \& Connor, 2017). Portanto, diante de um paciente em assistência paliativa, é fundamental a participação ativa da família no processo.

\section{Reabilitaçáo nos Cuidados Paliativos}

Os Cuidados Paliativos de Reabilitação são realizados a partir de equipes interdisciplinares para desenvolver planos de resposta não apenas aos aspectos fisiológicos, 
mas também às necessidades psicológicas, sociais e espirituais dos pacientes e de seus familiares/cuidadores (Franklin \& Cheville, 2015; Tiberini \& Richardson, 2015).

Entretanto, defendemos que a reabilitação seja compreendida e realizada de forma transdisciplinar, bem como intervençōes planejadas sobre os domínios do cuidado (físico, social, psicológico e espiritual) ao mesmo tempo. O fracionamento das açôes de reabilitação, ou demasiada ênfase em uma dimensão em detrimento das demais, favorece o potencial iatrogênico das açóes de reabilitação, não respeitando a complexidade do indivíduo e os avanços da ciência para melhora da qualidade de vida, funcionalidade global e dignidade da pessoa humana.

Em CP, a reabilitação deve buscar resultados multidimensionais que vão além dos que estão relacionados especificamente ao estado de doença, como a funcionalidade e parâmetros de qualidade de vida. Nesse sentido, estratégias de reabilitação contribuem para os $\mathrm{CP}$, mantendo e, se possível, promovendo a funcionalidade do paciente durante um período de declínio sistêmico esperado, além de fornecer caminhos para prevenir ou desacelerar complicaçóes deletérias, como descondicionamento generalizado, desagregação e contraturas musculares e aspectos relacionados a outras dimensóes do cuidado (Franklin \& Cheville, 2015; Tiberini \& Richardson, 2015).

Geralmente, são desenvolvidos planos de reabilitação baseados nos seis domínios funcionais para os escores da Medida de Independência Funcional: cuidados pessoais; controle esfincteriano (intestino e bexiga); comunicação; cognição social; interação social; e resolução de problemas e memória. Assim, a reabilitação em CP constitui uma integração contínua de serviços que deve iniciar concomitantemente ao diagnóstico e tratamento da doença. Pacientes em CP podem se beneficiar de intervençóes cuidadosamente selecionadas, projetadas para manter ou até mesmo restaurar elementos-chave de suas funçóes (Tiberini \& Richardson, 2015).

Nesse sentido, o atendimento às necessidades multidimensionais de pacientes, familiares e profissionais, bem como aquelas que emergem do próprio processo do cuidado é uma tarefa complexa e desafiadora. A ampliação do paradigma da saúde, bem como de concepçóes acerca do cuidado em saúde, apontam para a importância de conjugar as práticas de cuidado aos avanços da biomedicina e da tecnologia. A incorporação de novos métodos e técnicas ao cuidado pode servir como facilitador ao grande desafio de consolidar vínculos entre profissionais e pacientes e produzir cuidado com base em uma perspectiva ampliada e multidimensional, visualizando o cuidado em saúde como processo em ato, mas também como acontecimento, fruto da construção idiossincrática inerente a cada encontro.

Assim, a RV se evidencia como uma ferramenta que possibilita intervençóes de forma íntegra e segura, associada ou não ao tratamento padrão, potencializando e atingindo aspectos que a terapia convencional muitas vezes, por si só, não consegue. Além disso, a RVI pode ser utilizada para intervençóes em diversas áreas do conhecimento, bem como para aspectos relativos à saúde e seus domínios físico, social, psicológico e espiritual. Assim, defendemos a incorporação da RV nos projetos terapêuticos de reabilitaçáo, pautada no conceito de Dor Total, o que amplia a visão profissional referente ao cuidado holístico aos pacientes e familiares.

A reabilitação deve ser compreendida e trabalhada da maneira mais ampla, desde seu aspecto conceitual até as suas consideraçóes técnicas e instrumentais. Apoiando-nos no incrível potencial evidenciado pela TERV, apresentamos uma proposta conceitual para 
reabilitação, coerente com os avanços tecnológicos e evidências de melhores práticas em saúde, baseadas no cuidado humanizado, centrado na pessoa e sensível às dimensóes da Dor Total.

\section{Reabilitação Total: uma Proposta Conceitual}

Frente aos benefícios e inovaçóes evidenciados pela incorporação da RVI ao campo da saúde e da reabilitação, questionamos incorporaçóes ao conceito atual de reabilitação e apresentamos o conceito de Reabilitação Total, visando a uma reabilitação pautada no trabalho holístico orientado para preservação, ganho e/ou manutenção da funcionalidade global e dignidade do paciente e de seus familiares, que enfrentam doenças e/ou condiçóes ameaçadoras a sua integridade física, psicológica, social e/ou espiritual, bem como para a melhora da saúde e qualidade de vida, através de ambientes físicos e/ou virtuais, com intervenções orientadas para as dimensóes físicas, psicológicas, sociais e espirituais, cujo projeto terapêutico seja construído e conduzido sob a perspectiva da inter ou transdisciplinaridade e intersetorialidade.

Sob a perspectiva da Reabilitação Total, evidenciam-se novas possibilidades terapêuticas e inovaçóes, com intervençóes passíveis de aprimoramento e/ou descobertas, ampliando a oferta terapêutica para melhora da saúde, reabilitação e qualidade de vida de pacientes, familiares e equipe envolvida no cuidado e apoio.

Assim, a mudança no modo de vida e relacionamento dos indivíduos com novas tecnologias, em particular a RV, bem como sua incorporação como ferramenta terapêutica, pode se desdobrar em uma transformação do paradigma biomédico através não só da incorporação de novos métodos, técnicas e instrumentos de cuidado, como também da projeção, grau de importância e priorização que estes métodos poderão adquirir no universo do cuidado em saúde no futuro.

Dessa forma, esse conceito cunhado pelos autores permite ampliação da visão dos profissionais de saúde - e usuários - frente às possibilidades terapêuticas e reabilitativas. A partir dele, expande-se o objeto de atenção da reabilitação para além da dimensão física, incluindo as dimensóes psicológicas, sociais e espirituais como inerentes aos processos de vida e adoecimento e, portanto, necessárias de ponderação nos planos terapêuticos. Além disso, apresenta um horizonte de açôes que mesclem intervençóes padróes e virtuais ou mesmo realizadas totalmente em ambientes virtuais.

Isso, por sua vez, demanda formação e especialização profissional no âmbito da reabilitação em ambiente virtual, o que ainda não é realizado e merece atenção especial pelas Instituiçóes de Ensino Superior e diretrizes curriculares em saúde. Essa perspectiva extrapola o que encontramos hoje nas formaçôes acadêmicas em saúde; porém, denota alto grau de atualização e orientação pelas melhores práticas evidenciadas pela Medicina Baseada em Evidências.

\section{Consideraçóes Finais}

Este manuscrito apresenta um processo reflexivo acerca de novas tecnologias e o potencial da Realidade Virtual no campo da saúde e inicia discussão pela ampliação de concepçóes acerca da reabilitação nas diferentes dimensóes - física, psicológica, social e 
espiritual - com vistas a potencializar caminhos e resultados em saúde por meio da Realidade Virtual Imersiva.

Apoiados nessa perspectiva, propomos o conceito de Reabilitação Total. Acreditamos que, de acordo com a base contextual apresentada, essa perspectiva é cercada de plausibilidade científica e metafísica; permite-se, assim, a reflexão e ampliação da visão dos profissionais de saúde e usuários frente a novas perspectivas para a promoção da saúde, além da prevenção, recuperação e intervenções de reabilitação.

Além disso, ressaltamos a potência da Realidade Virtual Imersiva não apenas como possibilidade terapêutica concomitante a métodos tradicionais de reabilitação, mas também para uma possível dimensão de cuidado e intervençóes realizadas totalmente em ambientes virtuais, o que, por sua vez, evidencia uma demanda por profissionais especializados em reabilitação virtual.

Claro que essa perspectiva vai além do que encontramos atualmente nas formaçóes acadêmicas dos profissionais de saúde. Porém, se lembrarmos que há algumas décadas nem imaginávamos que a RVI chegaria a tal visibilidade e utilizaçáo nos processos terapêuticos em saúde, o que impede que a RVI e seus aprimoramentos contribua para uma revolução na clínica e nos modos de pensar e operacionalizar o cuidado em saúde?

É possível perceber o início de uma nova era em que novas possibilidades terapêuticas se desenham. Assim, a Realidade Virtual Imersiva se apresenta como um elemento revolucionário no futuro da saúde, em particular na reabilitação. O futuro é agora!

\section{Referências}

Ahmadpour, N., Randall, H., Choksi, H., Gao, A., Vaughan, C., \& Poronnik, P. (2019). Virtual reality interventions for acute and chronic pain management. The International Journal of Biochemistry \& Cell Biology, 114, 105568. PMid:31306747. http://dx.doi.org/10.1016/j.biocel.2019.105568.

Anderson, A. P., Mayer, M. D., Fellows, A. M., Cowan, D. R., Hegel, M. T., \& Buckey, J. C. (2017). Relaxation with immersive natural scenes presented using virtual reality. Aerospace Medicine and Human Performance, 88(6), 520-526. PMid:28539139. http://dx.doi.org/10.3357/AMHP.4747.2017.

Blascovich, J., \& Bailenson, J. (2011). Infinite reality: avatars, eternal life, new worlds, and the dawn of the virtual revolution. New York: HarperCollins.

Buyuk, E. T., Odabasoglu, E., Uzsen, H., \& Koyun, M. (2021). The effect of virtual reality on Children's anxiety, fear, and pain levels before circumcision. Journal of Pediatric Urology, 17(4), 567.e1-567.e8. PMid:34006462. http://dx.doi.org/10.1016/j.jpurol.2021.04.008.

Cho, D. R., \& Lee, S. H. (2019). Effects of virtual reality immersive training with computerized cognitive training on cognitive function and activities of daily living performance in patients with acute stage stroke: a preliminary randomized controlled trial. Medicine, 98(11), e14752. PMid:30882644. http://dx.doi.org/10.1097/MD.0000000000014752.

Cipresso, P., Giglioli, I. A. C., Raya, M. A., \& Riva, G. (2018). The past, present, and future of virtual and augmented reality research: a network and cluster analysis of the literature. Frontiers in Psychology, 9, 2086. PMid:30459681. http://dx.doi.org/10.3389/fpsyg.2018.02086.

Cortés-Pérez, I., Nieto-Escamez, F. A., \& Obrero-Gaitán, E. (2020). Immersive virtual reality in stroke patients as a new approach for reducing postural disabilities and falls risk: a case series. Brain Sciences, 10(5), 296. PMid:32429085. http://dx.doi.org/10.3390/brainsci10050296.

Felnhofer, A., Hlavacs, H., Beutl, L., Kryspin-Exner, I., \& Kothgassner, O. D. (2019). Physical presence, social presence, and anxiety in participants with social anxiety disorder during virtual cue exposure. Cyberpsychology, Behavior, and Social Networking, 22(1), 46-50. PMid:30407091. http://dx.doi.org/10.1089/cyber.2018.0221. 
Ferguson, C., Shade, M. Y., Blaskewicz Boron, J., Lyden, E., \& Manley, N. A. (2020). Virtual reality for therapeutic recreation in dementia hospice care: a feasibility study. The American Journal of Hospice \& Palliative Care, 37(10), 809-815. PMid:31975609. http://dx.doi.org/10.1177/1049909120901525.

Franklin, D. J., \& Cheville, A. L. (2015). Medical rehabilitation and the palliative care patient. In N. Cherny, M. Fallon, S. Kaasa, R. Portenoy \& D. C. Currow (Eds.), Oxford textbook of palliative medicine (pp. 1-31). Oxford: Oxford University Press.

Gerardi, M., Cukor, J., Difede, J., Rizzo, A., \& Rothbaum, B. O. (2010). Virtual reality exposure therapy for post-traumatic stress disorder and other anxiety disorders. Current Psychiatry Reports, 12(4), 298305. PMid:20535592. http://dx.doi.org/10.1007/s11920-010-0128-4.

Gerig, N., Mayo, J., Baur, K., Wittmann, F., Riener, R., \& Wolf, P. (2018). Missing depth cues in virtual reality limit performance and quality of three dimensional reaching movements. PLoS One, 13(1), e0189275. PMid:29293512. http://dx.doi.org/10.1371/journal.pone.0189275.

Gómez-Batiste, X., \& Connor, S. (2017). Building integrated palliative care programs and services. Londres: Worldwide Hospice Palliative Care Alliance.

Hsieh, W. T. (2020). Virtual reality video promotes effectiveness in advance care planning. BMC Palliative Care, 19(1), 125. PMid:32799876. http://dx.doi.org/10.1186/s12904-020-00634-w.

Ioannou, A., Papastavrou, E., Avraamides, M. N., \& Charalambous, A. (2020). Virtual reality and symptoms management of anxiety, depression, fatigue, and pain: a systematic review. SAGE Open Nursing, 6, 1-13. PMid:33415290. http://dx.doi.org/10.1177/2377960820936163.

Izard, S. G., Juanes, J. A., García Peñalvo, F. J., Estella, J. M. G., Ledesma, M. J. S., \& Ruisoto, P. (2018). Virtual reality as an educational and training tool for medicine. Journal of Medical Systems, 42(3), 50. PMid:29392522. http://dx.doi.org/10.1007/s10916-018-0900-2.

Jang, S. H., You, S. H., Hallett, M., Cho, Y. W., Park, C. M., Cho, S. H., Lee, H. Y., \& Kim, T. H. (2005). Cortical reorganization and associated functional motor recovery after virtual reality in patients with chronic stroke: an experimenter-blind preliminary study. Archives of Physical Medicine and Rehabilitation, 86(11), 2218-2223. PMid:16271575. http://dx.doi.org/10.1016/j.apmr.2005.04.015.

Johnson, L., Bird, M. L., Muthalib, M., \& Teo, W. P. (2018). Innovative STRoke Interactive Virtual thErapy (STRIVE) online platform for community-dwelling stroke survivors: a randomised controlled trial protocol. BMJ Open, 8(1), e018388. PMid:29317414. http://dx.doi.org/10.1136/bmjopen-2017-018388.

Johnson, T., Bauler, L., Vos, D., Hifko, A., Garg, P., Ahmed, M., \& Raphelson, M. (2020). Virtual reality use for symptom management in palliative care: a pilot study to assess user perceptions. Journal of Palliative Medicine, 23(9), 1233-1238. PMid:31895637. http://dx.doi.org/10.1089/jpm.2019.0411.

Kim, W. S., Cho, S., Ku, J., Kim, Y., Lee, K., Hwang, H. J., \& Paik, N. J. (2020). Clinical application of virtual reality for upper limb motor rehabilitation in stroke: review of technologies and clinical evidence. Journal of Clinical Medicine, 9(10), 3369. PMid:33096678. http://dx.doi.org/10.3390/jcm9103369.

Kim, W. S., Cho, S., Park, S. H., Lee, J. Y., Kwon, S., \& Paik, N. J. (2018). A low cost kinect-based virtual rehabilitation system for inpatient rehabilitation of the upper limb in patients with subacute stroke: a randomized, double-blind, sham-controlled pilot trial. Medicine, 97(25), e11173. PMid:29924029. http://dx.doi.org/10.1097/MD.0000000000011173.

Lee, A. L., DeBest, M., Koeniger-Donohue, R., Strowman, S. R., \& Mitchell, S. E. (2020). The feasibility and acceptability of using virtual world technology for interprofessional education in palliative care: a mixed methods study. Journal of Interprofessional Care, 34(4), 461-471. PMid:31431115. http://dx.doi.org/10.1080/13561820.2019.1643832.

Lee, S. Y., \& Kang, J. (2020). Effect of virtual reality meditation on sleep quality of intensive care unit patients: a randomised controlled trial. Intensive \& Critical Care Nursing, 59, 102849. PMid:32241625. http://dx.doi.org/10.1016/j.iccn.2020.102849.

Matthews, D. (2018). Virtual-reality applications give science a new dimension. Nature, 557(7703), 127 128. PMid:29713071. http://dx.doi.org/10.1038/d41586-018-04997-2.

Miccinesi, G., Caraceni, A., Garetto, F., Zaninetta, G., Bertè, R., Broglia, C. M., Farci, B., Aprile, P. L., Luzzani, M., Marzi, A. M., Mercadante, S., Montanari, L., Moroni, M., Piazza, E., Pittureri, C., 
Tassinari, D., Trentin, L., Turriziani, A., Zagonel, V., \& Maltoni, M. (2020). The path of cicely saunders: the "peculiar beauty" of palliative care. Journal of Palliative Care, 35(1), 3-7. PMid:30871411. http://dx.doi.org/10.1177/0825859719833659.

Montana, J. I., Tuena, C., Serino, S., Cipresso, P., \& Riva, G. (2019). Neurorehabilitation of spatial memory using virtual environments: a systematic review. Journal of Clinical Medicine, 8(10), 1516. PMid:31547137. http://dx.doi.org/10.3390/jcm8101516.

Murdoch, M., \& Davies, J. (2017). Spiritual and affective responses to a physical church and corresponding virtual model. Cyberpsychology, Behavior, and Social Networking, 20(11), 702-708. PMid:29072960. http://dx.doi.org/10.1089/cyber.2017.0249.

Nwosu, A. C., Mills, M., Roughneen, S., Stanley, S., Chapman, L., \& Mason, S. R. (2021). Virtual reality in specialist palliative care: a feasibility study to enable clinical practice adoption. BMJ Supportive \& Palliative Care, 0, 1-5. PMid:33597168. http://dx.doi.org/10.1136/bmjspcare-2020-002327.

Pardo, P. J., Suero, M. I., \& Pérez, Á. L. (2018). Correlation between perception of color, shadows, and surface textures and the realism of a scene in virtual reality. Journal of the Optical Society of America, 35(4), 130-135. PMid:29603966. http://dx.doi.org/10.1364/JOSAA.35.00B130.

Perna, M. (2021). The potential of personalized virtual reality in palliative care: a feasibility trial. The American Journal of Hospice \& Palliative Care, 38(12), 1-7. PMid:33583203. http://dx.doi.org/10.1177/1049909121994299.

Qian, J., McDonough, D. J., \& Gao, Z. (2020). The effectiveness of virtual reality exercise on individual's physiological, psychological and rehabilitative outcomes: a systematic review. International Journal of Environmental Research and Public Health, 17(11), 4133. PMid:32531906. http://dx.doi.org/10.3390/ijerph17114133.

Russo, M., De Luca, R., Naro, A., Sciarrone, F., Aragona, B., Silvestri, G., Manuli, A., Bramanti, A., Casella, C., Bramanti, P., \& Calabrò, R. S. (2017). Does body shadow improve the efficacy of virtual reality-based training with BTS NIRVANA?: a pilot study. Medicine, 96(38), e8096. PMid:28930852. http://dx.doi.org/10.1097/MD.0000000000008096.

Tiberini, R., \& Richardson, H. (2015). Rehabilitative Palliative Care: A Challenge for the 21st Century. UK: Hospice UK.

Tieri, G., Morone, G., Paolucci, S., \& Iosa, M. (2018). Virtual Reality in Cognitive and Motor Rehabilitation: Facts, Fiction and Fallacies. Expert Review of Medical Devices, 15(2), 107-117. PMid:29313388. http://dx.doi.org/10.1080/17434440.2018.1425613.

Vaquero-Blasco, M. A., Perez-Valero, E., Morillas, C., \& Lopez-Gordo, M. A. (2021). Virtual reality customized 360-degree experiences for stress relief. Sensors, 21(6), 1-15. PMid:33810135. http://dx.doi.org/10.3390/s21062219.

Wang, S. S. Y., Teo, W. Z. W., Teo, W. Z. Y., \& Chai, Y. W. (2020). Virtual reality as a bridge in palliative care during COVID-19. Journal of Palliative Medicine, 23(6), 756. PMid:32324080. http://dx.doi.org/10.1089/jpm.2020.0212.

Weber, S., Weibel, D., \& Mast, F. W. (2021). How to get there when you are there already? Defining presence in virtual reality and the importance of perceived realism. Frontiers in Psychology, 12, 628298. PMid:34025504. http://dx.doi.org/10.3389/fpsyg.2021.628298.

Wijma, E. M., Veerbeek, M. A., Prins, M., Pot, A. M., \& Willemse, B. M. (2018). A virtual reality intervention to improve the understanding and empathy for people with dementia in informal caregivers: results of a pilot study. Aging \& Mental Health, 22(9), 1115-1129. PMid:28691861. http://dx.doi.org/10.1080/13607863.2017.1348470.

World Health Organization - WHO. (2018). Integrating palliative care and symptom relief into primary health care: $A$ WHO guide for planners, implementers and managers. Genebra: WHO.

Zhu, S., Sui, Y., Shen, Y., Zhu, Y., Ali, N., Guo, C., \& Wang, T. (2021). Effects of virtual reality intervention on cognition and motor function in older adults with mild cognitive impairment or dementia: a systematic review and meta-analysis. Frontiers in Aging Neuroscience, 13, 586999. PMid:34025384. http://dx.doi.org/10.3389/fnagi.2021.586999. 


\section{Contribuiçáo dos Autores}

Breno Augusto Bormann de Souza Filho e Érika

Fernandes Tritany foram igualmente responsáveis pela concepção do manuscrito, com participação efetiva no processo de análise, interpretação dos dados, redação e revisão crítica do conteúdo. Todos os autores aprovaram a versão final do texto.

\section{Autor para correspondência}

Breno Augusto Bormann de Souza Filho

e-mail: brenobormann@hotmail.com

\section{Editora de seçáo}

Profa. Dra. Iza Faria-Fortini 